\title{
IDOSOS ATENDIDOS EM UNIDADE BÁSICA DE SAÚDE: EXPERIÊNCIAS SOBRE POLIMEDICAÇÃO
}

\section{ELDERLY ATTENDED IN THE BASIC HEALTH UNIT: EXPERIENCES ON POLYMEDICATION}

\author{
Cathia Valadão Silva ${ }^{1}$ \\ Elza de Fátima Ribeiro Higa ${ }^{2}$ \\ Maria José Sanches Marin ${ }^{3}$ \\ Flávia Cristina Goulart ${ }^{4}$ \\ Carlos Alberto Lazarini ${ }^{5}$
}

\begin{abstract}
Resumo: O envelhecimento acarreta múltiplas alterações e doenças, intensificando, assim, a necessidade de recursos de saúde. A literatura aponta que, nesta fase da vida, é frequente a polimedicação, caracterizada pelo uso de cinco ou mais medicamentos. Logo, objetivou-se compreender as experiências de idosos sobre o significado da polimedicação. Pesquisa com abordagem qualitativa, a partir de entrevistas com 14 idosos, atendidos em Unidade Básica de Saúde, as quais foram gravadas, transcritas e, posteriormente, submetidas à técnica de Análise de Conteúdo, modalidade temática. Na análise, emergiram três categorias temáticas: Saúde e longevidade, Quantidade excessiva, e Confiança no médico. Idosos usuários de Unidade Básica de Saúde, ao serem estimulados a refletir sobre o significado do uso diário de cinco ou mais medicamentos, expressaram experiências acerca da polimedicação.
\end{abstract}

Palavras-chave: Idosos; Polimedicação; Centros de Saúde.

Abstract: Aging brings multiple changes and diseases, thus intensifying the need for health resources. The literature indicates that, at this stage of life, polymedication is frequent, characterized by the use of five or more drugs. Therefore, it was aimed to understand the experiences of the elderly about the meaning of the polymedication. Research with a qualitative approach, based on interviews with 14 elderly people, attended at a Basic Health Unit, which were recorded, transcribed and later submitted to the Content Analysis technique, thematic modality. In the analysis, three thematic categories emerged: Health and longevity, Excessive Quantity, and Trust in the physician. Elderly users of the Basic Health Unit, when being stimulated to reflect on the meaning of the daily use of five or more medications, expressed experiences about the polymedication.

Keywords: Elderlies; Polypharmacy; Health Center.

\footnotetext{
${ }^{1}$ Mestre em Saúde e Envelhecimento pela Faculdade de Medicina de Marília, (Famema), Marília, SP, Brasil.E-mail: cathiavaladao@ hotmail.com

${ }^{2}$ Doutora em Enfermagem Fundamental pela Univerisidade de São Paulo (USP). Docente da disciplina de Educação em Ciências da Saúde da Faculdade de Medicina de Marília (Famema), Marília, SP, Brasil. Email: hirifael@gmail.com

${ }^{3}$ Doutora em Enfermagem Fundamental pela Univerisidade de São Paulo (USP). Docente da disciplina de Fundamentos de Enfermagem da Faculdade de Medicina de Marília (Famema), Marilia, SP, Brasil. E-mail: marnadia@terra.com.br

${ }^{4}$ Doutora em Neurociência do Comportamento pelo Instituto de Psicologia da Univerrsidade de São Paulo (USP). Docente da disciplina de Farmacologia da Faculdade de Medicina de Marília (Famema), Marília, SP, Brasil.E-mail: flaviagoulart@usp.br

${ }^{5}$ Doutor em Ciências pelo Instituto de Biociência, Universidade de São Paulo (USP). Docente da disciplina de Farmacologia da Faculdade de Medicina de Marília (Famema), Marília, SP, Brasil. E-mail: carlos.lazarini@gmail.com
} 


\section{Introdução}

A população brasileira passa por processo acelerado de envelhecimento. Segundo o censo demográfico de 2010, essa população era de 190.755.199 milhões de pessoas, sendo aproximadamente 17,6 milhões de idosos (BRASIL, 2018). O aumento significativo do número de anos vividos ocasiona mudanças na estrutura etária da população. O segmento de 80 anos ou mais, no Brasil, é o que registra maior crescimento. Em 2000, esse segmento representava 12\% do total de idosos e, em 2010, 15,3\% (BRASIL, 2016).

O processo de envelhecimento, na maioria dos casos, vem acompanhado da intensificação de Doenças Crônicas Não Transmissíveis (DCNT), fenômeno, também, caracterizado no Brasil, segundo a Pesquisa Nacional de Saúde (PNS), de 2013 (IBGE, 2014). O envelhecimento acarreta múltiplas alterações e doenças, elevando, assim, a necessidade de recursos de saúde, destacando-se o uso de medicamentos (SILVA; MACEDO, 2013).

Em torno de $80 \%$ dos idosos padecem de uma ou mais DCNT (MUNIZ et al., 2017) e $36 \%$ podem padecer de três delas (SILVA; SCHIMIDT; SILVA, 2012). As mudanças orgânicas decorrentes do envelhecimento interferem na resposta aos medicamentos, mesmo assim, o modelo de cuidado à pessoa idosa no Brasil, ainda, apresenta-se, no fazer prático, centrado no paradigma curativo, ou seja, predominando o tratamento pontual de doenças por meio da farmacoterapia (MUNIZ et al., 2017).

Entre os idosos ocorre com frequência a utilização de vários medicamentos, simultaneamente. Estudo de base populacional, com amostra composta por 1.705 idosos, demonstrou prevalência de $32 \%$ dos entrevistados utilizando cinco ou mais medicamentos (PEREIRA et al., 2017), caracterizando a polimedicação, prática definida pelo uso de cinco ou mais medicamentos prescritos, ou não, em qualquer momento, ou suplementos naturais (CASTRO-RODRIGUEZ; OROZCO-HERNÁNDEZ; MARINMEDINA, 2015).

Outro estudo realizado com 110 idosos atendidos em Unidade de Atenção Primária à Saúde de município do interior de Minas Gerais, Brasil, sendo 74\% do sexo feminino, idade média de 69,8 anos e 30,9\% sem escolaridade, encontrou que a prática de polimedicação está presente em 40\% destes (PAULA JÚNIOR et al., 2013).

Alguns dos dados anteriores se assemelham aos encontrados em pesquisa com 299 idosos no Centro de Convivência do Idoso, da Associação Municipal de Apoio 
Comunitário, da Prefeitura Municipal de Juiz de Fora, em que dos entrevistados, 71,6\% eram do sexo feminino, com média de idade de 72,7 anos, 74,6\% concluíram o ensino fundamental e $25,1 \%$ do total de entrevistados faziam uso concomitante de cinco ou mais fármacos (CHEHUEN NETO et al., 2011).

Em relação à prática de polimedicação declarada por idosos, foram encontradas para faixa etária entre 60 e 69 anos as porcentagens: 25,5\%, 15,8\%, 38,3\%, 20,5\% (PEREIRA et al., 2017; RAMOS et al., 2016; SANTOS et al., 2013; SALES; SALES; CASOTTI, 2017); na faixa etária entre 70 e 79 anos, observaram-se: 37,3\%, 21,5\%, 27,3\%, 38,5\% (PEREIRA et al., 2017; RAMOS et al., 2016; SANTOS et al., 2013; SALES; SALES; CASOTTI, 2017); e para os idosos com 80 anos ou mais: 42,0\%, 19,5\%, 35,4\%, 29,4\% (PEREIRA et al., 2017; RAMOS et al., 2016; SANTOS et al., 2013; SALES; SALES; CASOTTI, 2017). Esses resultados não permitem caracterizar faixa etária com predomínio da prática de polimedicação.

No que se refere a estudos de natureza qualitativa, os quais permitem levar a compreensão das variáveis que permeiam o uso da polimedicação por idosos, em Unidades Básicas de Saúde, ainda, são escassos. Todavia, pesquisas desta natureza podem contribuir para retratar as experiências desta faixa etária nesse contexto.

Arruda, Lima e Renovato (2013) encontraram que homens idosos podem seguir ou não a prescrição que lhes é determinada pelo médico, além de poder modificá-la, conforme concepções e necessidades, o que perpassa por questões importantes, como a medicalização do corpo masculino, a obrigação do uso, a não aceitação e a susceptibilidade atrelada à polimedicação.

Nesse sentido, estudo que objetivou compreender as representações sociais de idosos usuários do Sistema Único de Saúde, de município do interior do estado de São Paulo, Brasil, demonstrou a necessidade dos entrevistados em fazer uso irregular da medicação, o qual remete a não adesão ao tratamento, entendida enquanto uso da autonomia, de poder decisório de idosos sobre as próprias vidas, em relação ao que lhes fazem bem ou mal (SILVA et al., 2018).

Outro estudo de natureza qualitativa, realizado na Suécia, com 59 idosos, distribuídos em doze grupos focais, usando cinco ou mais medicamentos, demonstrou, inicialmente, ausência de problemas com uso de múltiplos medicamentos e que a sensação de bem-estar pelos medicamentos os mantiveram vivos (MOEN et al., 2009). Esses resultados divergem dos encontrados por Silva e colaboradores (2018), nos quais os idosos trouxeram, enquanto significado do uso da polimedicação, a presença de efeitos 
adversos, uma vez que perceberam alterações fisiológicas, como desconforto gastrointestinal, sonolência e cansaço.

A dificuldade na aceitação da dependência dos medicamentos e os efeitos adversos também esteve presente nas falas dos idosos no trabalho realizado na Suécia, cujos autores mostraram que a percepção dos participantes sobre os medicamentos dependia da interação médico-paciente e dos cuidados do sistema de saúde para o sucesso do tratamento (MOEN et al., 2009).

Ao considerar a existência de grande quantidade de idosos que faz uso de múltiplos medicamentos, que este representa risco às condições de vida e saúde destes, que a maioria dos estudos existentes tem se limitado a abordar quantitativamente essa temática, a presente pesquisa partiu da seguinte inquietação: quais significados atribuídos por idosos às experiências com uso da polimedicação? Assim, objetivo-se compreender as experiências de idosos sobre o significado da polimedicação.

\section{Metodologia}

Pesquisa qualitativa, com abordagem exploratória, transversal, de campo e interpretada pela técnica de análise de conteúdo, a qual faz parte das metodologias utilizadas na investigação social para análise e interpretação de dados obtidos (AMADO; COSTA; CRUSOÉ, 2014).

A pesquisa foi realizada com 14 idosos frequentadores de Unidade Básica de Saúde (UBS), órgão de saúde do município e responsável pelo monitoramento e pelas ações primárias e secundárias de saúde para região, localizada em município do interior do Estado de São Paulo, Brasil. O município possuía população estimada para o ano de 2017 de 65.758 habitantes, da qual 17,1\% eram idosos (IBGE, 2017). A UBS selecionada foi aquela que concentra a maior porcentagem de idosos em relação ao total de habitatnes. A população da área de abrangência dessa UBS compreende total de, aproximadamente, 8.000 pessoas, destas, cerca de $22,5 \%$ são idosos.

A amostra foi intencional, obtida por conveniência. A pesquisa foi realizada por meio de entrevistas com idosos, de ambos os sexos. Antes do início da entrevista, foi verificada a capacidade cognitiva e de comunicação do idoso para compreender a pergunta e fornecer informações necessárias ao objeto da pesquisa, o que foi verificado por meio da aplicação do Mini Exame do Estado Mental (MEEM) (ALMEIDA, 1998). 
O MEEM é um teste neuropsicológico que avalia a função cognitiva, por meio da abordagem dos domínios: orientação temporal e espacial, memória imediata e de evocação, cálculo, linguagem, repetição, compreensão e escrita. $\mathrm{Na}$ validação e adaptação à população brasileira, as notas de corte estabelecidas foram: 19 para analfabetos; 23 para aqueles que têm entre 1 e 3 anos de escolaridade; 24, para aqueles com 4 a 7 anos de escolaridade; e 28, acima de sete anos de escolaridade.

Foram incluídos idosos (60 anos ou mais) que faziam uso contínuo de polimedicação. Este dado foi obtido a partir de consulta ao prontuário, ocasião em que também se obteviveram endereço e telefone do idoso. Inicialmente, foi realizado contato telefônico para informar sobre a pesquisa e marcar horário para realização da coleta. $\mathrm{Na}$ sequência, essa informação foi confirmada com o próprio idoso, a partir do seguinte questionamento: quantos medicamentos o senhor(a) utiliza por dia? Como critério de exclusão, foram considerados: três visitas consecutivas com ausência do idoso no domicílio, impossibilidade do idoso em responder à entrevista e mudança de endereço do idoso. Destaca-se que não houve recusa em participar da pesquisa, uma vez que os 14 primeiros idosos contactados atenderam aos critérios de inclusão.

Os dados foram obtidos por meio de entrevista realizada no domicílio do idoso, com o cuidado de mantê-lo em local reservado da casa. Para Minayo (2013), a entrevista é a estratégia mais utilizada em pesquisas de campo. É um encontro entre dois indivíduos com a finalidade de se obter informações acerca de determinado fenômeno (MARCONI; LAKATOS, 2010), mediante conversação, de iniciativa do pesquisador, que se destina a construir informações acerca do objeto de pesquisa e temas pertinentes (MINAYO, 2013). O período da coleta de dados foi entre janeiro e fevereiro de 2018.

As entrevistas realizadas contaram com uma questão aberta sobre o significado atribuído pelo idoso sobre o uso da polimedicação. As falas foram gravadas e transcritas na íntegra, para análise posterior.

Os dados obtidos foram interpretados por meio da técnica de Análise de Conteúdo, modalidade temática que se constitui por três etapas: pré-análise, exploração do material e interpretação. Na pré-análise, estrutura-se o copus da pesquisa que deve ser organizado a partir das regras de: pertinência, na qual inclui o quadro teórico e a fonte de informação deve ser adequada ao objetivo da pesquisa; representatividade, que aponta a importância da amostra analisada; objetividade e fidedignidade, para que não se sobreponha a subjetividade do pesquisador durante a interpretação dos dados obtidos (AMADO; COSTA; CRUSOÉ, 2014; BARDIN, 2016). 
$\mathrm{Na}$ segunda etapa de exploração do material, é realizada a categorização por meio da elaboração das unidades de análise, que podem ser palavras, frases, expressões significativas, personagem e temas relevantes. A exploração do material visa alcançar o núcleo de compreensão ou de sentido do texto. A escolha das categorias é uma intervenção sobre a mensagem com critérios definidos, coerentes com o objeto de estudo, o problema de pesquisa, os objetivos e a fundamentação teórica.

As categorias de análise são estruturadas por meio dos seguintes critérios: exaustão, que indicam a importância de inclusão dos dados obtidos; exclusão mútua, na qual cada elemento não pode existir em mais de uma categoria; homogeneidade, que permite a organização, de um mesmo conjunto de categorias, com os mesmos critérios; concretude, as considerações sobre o fenômeno estudado não devem serem expressas de forma abstrata, devido à multiplicidade de significados; e produtividade, na qual as inferências proporcionam novos resultados e novas compreensões (AMADO; COSTA; CRUSOÉ, 2014; BARDIN, 2016).

A terceira etapa interpretativa procura a análise arraigada e expandida sobre os conteúdos evidentes e ocultos do conjunto do material. Neste processo, os dados obtidos são relacionados às estruturas semânticas e sociológicas, tendo em vista a articulação dos fatores decisivos em suas características. Assim, a interpretação emerge da síntese entre as demandas da pesquisa, dos resultados obtidos, a partir da análise e das perspectivas teóricas adotadas (AMADO; COSTA; CRUSOÉ, 2014; BARDIN, 2016).

O estudo foi aprovado pelo Comitê de Ética em Pesquisa, da Faculdade de Medicina de Marília, conforme CAAE nº 69897017.6.0000.5413, em 11/12/17. Os participantes assinaram o Termo de Consentimento Livre e Esclarecido (TCLE), antes da coleta de dados, como preconiza a Lei 510/2016 (BRASIL, 2016). Para apresentação dos resultados, os idosos foram codificados por meio da letra I, seguida de ordem numérica crescente I1 a I14, no sentido de garantir o anonimato dos participantes.

\section{Resultados e Discussão}

Os dados obtidos nesta pesquisa, a partir da análise das respostas à pergunta: "o que significa para o senhor(a) tomar essa quantidade de medicamentos todos os dias?", permitiram a elaboração de três categorias temáticas: Saúde e longevidade, Quantidade excessiva, e Confiança no médico. 


\subsection{Saúde e longevidade}

Os idosos entrevistados apresentaram a experiência dos medicamentos enquanto agentes capazes de restaurarem ou promoverem saúde e necessários às atuais condições de vida.

"Eu me sinto bem. Então, para ser sincera, eu já me acostumei por que eu tomo desde os meus 17 anos de idade. Eu procuro com isso tentar viver um pouco mais, se eu posso viver mais dez anos por que viver só cinco?”(I2).

O hábito de uso diário da medicação remete à adesão eficaz ao tratamento. Estudo realizado com 165 idosos de município do interior do Ceará, Brasil, mostrou que a maioria dos entrevistados afirmou que não esquece de tomar os medicamentos diariamente e que, além disso, recebeu orientações sobre as indicações e os efeitos adversos dos medicamentos de que fazia uso (BEZERRA; BRITO; COSTA, 2016). Esses autores sugerem que este comprometimento do idoso quanto ao uso diário da medicação pode estar relacionado à assistência satisfatória de saúde por parte da equipe, com esclarecimentos resolutivos sobre a terapêutica medicamentosa de cada indivíduo.

Além disso, vivenciavam o uso diário de cinco ou mais medicamentos como necessários para que pudessem garantir sensação de bem-estar.

"Eu sinto alívio, fico bem. Se eu não tomar, eu fico doente. Se existem esses remédios que me ajudam, porque não vou tomar?”(I3).

"Eu preciso tomar de uns tempos para cá, porque atacou a pressão. Eu tomo porque é preciso e me sinto bem assim. Acabei me acostumando com isso." (I10).

A sensação de bem-estar referida pelos idosos ao utilizarem cinco ou mais medicamentos pode ser associada à autoavaliação de saúde positiva. Este resultado se contrapõe ao apresentado na literatura. Estudo com 9.019 idosos, distribuídos nas cinco regiões do país, mostrou que a prática de polimedicação esteve associada à autoavaliação de saúde ruim ou muito ruim (RAMOS et al., 2016). Já Borba e colaboradores (2018), ao avaliarem a adesão ao uso de medicamentos por idosos diabéticos, apontaram que o idoso que autopercebe a saúde como regular, desempenha maior rotina de cuidados com a doença, ou seja, mesmo não considerando a saúde como positiva, mantém o hábito diário do uso dos medicamentos.

Associado ao hábito diário e à garantia de sensação de bem-estar, o uso de polimedicação também foi relatado enquando necessário para sobrevivência.

"É minha sobrevivência, porque se eu não tomar, ou toma ou morre. Já me acostumei e tenho que conviver com eles. Eu tomo porque eu necessito e 
porque quero viver mais. Tem que tomar o medicamento certo, então, eu tomo" (I7).

"Tenho vontade de parar tudo, mas se eu não tomar, eu morro" (I4).

A percepção de que a quantidade de medicamentos está diretamente relacionada com a melhora dos sintomas e, por isso, permitem se acostumar com o uso diário destes, também esteve presente.

"Eu tomo porque é preciso e me sinto bem assim. Acabei me acostumando com isso." (I12).

Para muitos idosos, o medicamento se caracteriza como solução necessária para vida (ARRUDA; LIMA; RENOVATO, 2013). Esses autores observaram que a ingestão diária de um ou mais medicamentos fazia parte do cotidiano da vida dos idosos entrevistados, uma vez que os medicamentos propiciavam condições de se estabelecer a normalidade do corpo frente a situações de adoecimento.

A prática diária de usar medicamentos manifestava-se como importante aspecto na vida dos idosos entrevistados, tendo em vista que, em muitos casos, a terapia medicamentosa ocupa eixo central no cuidado à saúde (OLIVEIRA; SANTOS, 2016a).

\subsection{Quantidade excessiva}

O uso diário de cinco ou mais medicamentos (polimedicação) se constitui em quantidade exagerada. Isso pode ser constatado na fala do I6.

\footnotetext{
"Não gostaria que fossem tantos remédios assim, mas sou obrigada a tomar. Para mim, é um problemão, uma bela de uma porcaria. Tem hora que parece que a gente vai intoxicar de tomar tantos remédios. É droga bruta e, infelizmente, eu necessito, mas se eu pudesse, eu parava ontem. Tenho vontade de parar tudo" (I6).
}

Para Souza e colaboradores (2011), quando o idoso se depara com prescrição de medicamentos para uso prolongado, ou seja, uso contínuo em função do caráter crônico de doenças, a possibilidade de abandono da terapêutica ou mesmo a alteração desta, pode estar presente, como evidenciado enquanto desejo do I6. O uso contínuo de medicamentos requer, por parte do idoso, mudança e nova adaptação de rotinas para cumprimento da terapêutica instituída.

Possível estratégia para melhor entendimento do idoso acerca da necessidade do uso contínuo da medicação seria a implantação de ações de educação em saúde. Para tal, faz-se necessário que os profissionais de saúde forneçam informações de maneira simples e clara, que respeite a compreensão e os diferentes níveis educacionais de cada idoso. Neste sentido, estudo que objetivou explorar a experiência da alta hospitalar de idosos e 
respectivos familiares quanto ao manejo no uso de medicamentos, demonstrou comunicação inadequada entre profissionais e usuários, fato que pode dificultar o cumprimento do regime terapêutico (KNIGHT et al., 2013).

Cunha e colaboradores (2012) apontaram a preocupação de profissionais da Estratégia Saúde da Família acerca dos pacientes e o uso que estes faziam dos medicamentos, pois as equipes acreditavam que os usuários não compreendiam as orientações fornecidas e, muitas vezes, acabavam utilizando os fármacos de forma irracional, ficando expostos a vários riscos.

O núcleo de sentido anteriormente apresentado na fala do participante também esteve relacionado às estratégias de negação da rotina de uso de polimedicação. Muitos idosos passam a apresentar, ao longo do processo de envelhecimento, percepção que o associa à perda da saúde e juventude, como se envelhecer fosse sinônimo de adoecer.

\footnotetext{
"Seria tão bom não tomar remédio nenhum, porque se a gente não toma remédio, a gente tem saúde e saúde é tudo. Tenho saudade de quando eu não precisava tomar tudo isso. Eu fico muito triste e revoltada porque até outro dia eu me vangloriava da saúde que eu tinha” (I11).
}

Tratar uma ou mais doenças crônicas implica sobrecarga para vida do idoso (OLIVEIRA; SANTOS, 2016b), uma vez comprrende o envelhecimento pautado no aparecimento de doenças, imposição de limites para manutenção da vida, uso de medicamentos e perda da jovialidade (SILVA; MENANDRO, 2014)

Para Moraes e colaboradores (2016), os idosos demonstram o pensamento cartesiano e mecanicista, internalizado, relacionado ao saber biomédico, que compreende a velhice como equação matemática, na qual, de acordo com os autores, o "corpo velho = máquina defeituosa", ou seja, o sintoma representa o defeito que o idoso nomeia como "barulhinho" (MORAES et al., 2016, p. 323). Neste sentido, quando o idoso classifica a velhice como etapa da vida permeada de dificuldades físicas, propaga a concepção do corpo velho como máquina desgastada, na qual defeitos/doenças são naturais e esperados, mas, para os quais, a "oficina" da ciência biomédica não oferece "conserto", reforçando, uma vez mais, a compreensão de que "a velhice é doentia por si só" (MORAES et al., 2016, p. 323). 


\subsection{Confiança no médico}

Para os idosos, o saber biomédico é muito valorizado, especialmente na figura do médico, reconhecido como principal autoridade e detentor absoluto do conhecimento científico.

"É dado pelo médico e o médico dá conselhos para gente tomar esses medicamentos certinho e a gente toma e fica bem. Tem muita gente aí que não vai em médico, mas eu acompanho o médico" (I5).

"O médico receita, é a indicação do médico. Eu tomo por obediência a medicina! Se ele está indicando é porque é acertado! Então, eu estou tomando" (I9).

A soberania do papel do médico no tratamento medicamentoso é evidenciada em muitos estudos (OLIVEIRA; SANTOS, 2016b; FRIED; TINETTI; IANNONE, 2011). Isso parece estar atrelado a um comportamento de confiança absoluta do idoso na relação com o médico e os cuidados desse para consigo (WHILE et al., 2012), fato que faz com que sigam a terapia medicamentosa sem discutir ou questionar as prescrições (MORAES, 2012), inclusive a necessidade de continuidade do tratamento (ALVARENGA et al., 2015). Em contrapartida, o idoso pode depositar a autonomia de saúde no médico, à medida que a doença avança, e passa a acreditar que restam poucas opções para o tratamento, preferindo deixar a tomada de decisão a cargo do médico (FIELD et al., 2006).

Algumas pessoas acreditam que, à medida que a doença avança, restam poucas opções e, por isso, preferem transferir a decisão quanto ao tratamento somente ao médico, não buscando mais informações. Esta atitude pode representar preocupação ou ausência destas, quanto ao tratamento medicamentoso, sob três formas: aqueles que não sabem para que serve e/ou quais os efeitos colaterais do medicamento; pessoas que sabiam os nomes e os principais efeitos colaterais, e que contavam com os médicos para fornecer informações detalhadas; os que entendiam o diagnóstico e que estavam comprometidos em descobrir sempre algo mais (OLIVEIRA; SANTOS, 2016b).

$\mathrm{Na}$ visão do médico, o idoso apresenta alguns problemas em relação ao uso de medicamentos: em função do envelhecimento e com o prejuízo da memória, o idoso tende a esquecer de tomar o medicamento, conforme o prescrito; quanto ao número de medicamentos utilizados diariamente (polimedicação), o idoso, por ser atendido por diferentes médicos, em momentos distintos, acaba recebendo prescrições de medicamentos semelhantes, o que eleva ainda mais a quantidade diária a ser tomada; e, 
em função do anteriormente apresentado, o idoso se confunde, com os nomes dos medicamentos, inclusive com os dos genéricos (SILVA; SPINILLO, 2016).

Atrelado à confiança que o idoso deposita no profissional médico, está também a confiança na prescrição dos medicamentos e na forma de utilizá-los.

"O médico fala para tomar e eu tomo" (I1).

"Se o médico me passar um remédio, eu tomo certinho, porque quero melhorar" (14).

"Eu confio nos meus médicos que receitam esses remédios, então, eu acho que estou bem" (I8).

Em algumas realidades, ainda persiste relação de dominação entre médicos e idosos, caracterizada pelo pouco diálogo, pela linguagem científica pouco compreendida pelos pacientes ou mesmo pela grafia ilegível, fatores acarretam distanciamento e interações verticalizadas. Todavia, existem aqueles que se dizem respeitados e valorizados, o que favorece a comunicação, melhorando o entendimento sobre as doenças e reduzindo os riscos relacionados ao uso de medicamentos (OLIVEIRA; SANTOS, 2016b). Assim, a imagem do profissional médico é preservada, e a conduta deste, inquestionável e, como a argumentação de natureza biológica é apresentada com linguagem e técnicas complicadas, esse saber se apresenta como grande detentor do conhecimento sobre os processos da vida, o sofrimento e o adoecimento (MORAES et al., 2016).

\section{Considerações finais}

Idosos usuários de Unidade Básica de Saúde, ao serem estimulados a refletir sobre o significado do uso diário de cinco ou mais medicamentos, expressaram experiências acerca da polimedicação, as quais foram permeadas de diferentes opiniões, gerando, assim, três categorias temáticas.

A primeira sinalizou a importância do uso de medicamentos para manutenção e prolongamento de vida saudável. A segunda expressou a experiência e o incorformismo dos particianptes com a quantidade de medicamentos que necessitavam ingerir diariamente, e relacionaram este fato ao processo de envelhecimento. Na terceira categoria temática, apareceu com destaque a confiança que os idosos depositaram no médico e na prescrição que este fornecia, atribuindo-lhe a soberania intelectual em relação ao uso do medicamento. 
Identifica-se como limite para esta pesquisa o fato de retratar uma realidade local, com respectivas especificidades. No entando, os dados apresentados podem subsidiar as orientações a idosos acerca do uso de múltiplos medicamentos, de forma a considerar o significado atribuído por eles em seus cotidianos.

\section{Referências}

ALMEIDA, O. P. Mini exame do estado mental e o diagnóstico de demência no Brasil. Arq Neuro-Psiquiat, São Paulo, v. 56, n. 3B, p. 605-12, set. 1998.

ALVARENGA, J. M. et al. Uso de benzodiazepínicos entre idosos: o alívio de "jogar água no fogo", não pensar e dormir. Rev Bras Geriatr Gerontol., Rio de Janeiro, v. 18, n. 2, p. 249-258, 2015.

AMADO, J.; COSTA, A. P.; CRUSOÉ, N. A técnica da análise de conteúdo. In: AMADO, J. (coord.) Manual de investigação qualitativa em educação. 2. ed. Coimbra: Coimbra University Press, 2014. p. 301-355.

ARRUDA, G. O.; LIMA, S. C. S.; RENOVATO, R. D. Uso de medicamentos por homens idosos com polifarmácia: representações e práticas. Revista Latinoamericana de Enfermagem, Ribeirão Preto, v. 21, n. 6, p. 1337-44, nov./dez. 2013.

BARDIN, L. Análise de conteúdo. 3. reimp. da 1. ed. São Paulo: Edições 70, 2016.

BEZERRA, T. A., BRITO, M. A. A., COSTA, K. N. F. M. Caracterização do uso de medicamentos entre idosos atendidos em uma unidade básica de saúde da família. Cogitare Enferm., Curitiba, v. 21, n. 1, p. 01-11, jan./mar. 2016. Disponível em: https://revistas.ufpr.br/cogitare/article/view/43011/27630. Acesso em: 26 jan. 2019.

BORBA, A. K. O. T. et al. c. Ciência \& Saúde Coletiva, Rio de Janiero, v. 23, n. 3, p. 953-961, 2018.

BRASIL. Ministério da Saúde. Conselho Nacional de Saúde. Resolução no 510, de 7 de abril de 2016. Brasília, 2016. Disponível em:

http://bvsms.saude.gov.br/bvs/saudelegis/cns/2016/res0510_07_04_2016.html. Acesso em: 28 jan. 2019.

BRASIL. Instituto Brasileiro de Geografia e Estatística - IBGE. Número de idosos cresce $18 \%$ em 5 anos e ultrapassa 30 milhões em 2017. Rio de Janeiro, 2018. Disponível:

https://agenciadenoticias.ibge.gov.br/agencia-noticias/2012-agencia-de-noticias/noticias/20980numero-de-idosos-cresce-18-em-5-anos-e-ultrapassa-30-milhoes-em-2017. Acesso em: 19 nov. 2018.

CASTRO-RODRIGUEZ, A., OROZCO-HERNÁNDEZ, J. P., MARIN-MEDINA, D. S.

Polifarmácia y prescripción de medicamentos potencialmente no apropriados enancianos. Rev

Med Risaralda, Medellin, v. 21, n. 2, p. 52-57, 2015.

CHEHUEN NETO, J. A. et al. Uso de medicamentos por idosos de Juiz de Fora: um olhar sobre a polimedicação. HU Rev., Juiz de Fora, v. 37, n. 3, p. 305-13, jul./set. 2011 Disponível em: https://hurevista.ufjf.emnuvens.com.br/hurevista/article/view/1422. Acesso em: 04 jan. 2019. 
CUNHA, K. O. A. et al. Representações sobre uso racional de medicamentos em equipes da Estratégia Saúde da Família. Rev. Esc. Enferm., São Paulo, v. 46, n. 6, p. 1431-1437, 2012.

FIELD, K., ZIEBLAND, S., MCPHERSON, A., LEHMAN, R. "Can I come off the tablets now?" A qualitative analysis of heart failure patients' understanding of their medication. Fam Pract., Oxfor, v. 23, n. 6, p. 624-30, dec. 2006.

FRIED, T. R., TINETTI, M. E., IANNONE, L. Primary care clinicians' experiences with treatment decisionmaking for older persons with multiple conditions. Arch Intern Med., Chicago, v. 171, n. 1, p. 75-80, jan.. 2011.

IBGE. Pesquisa nacional de saúde: 2013: percepção do estado de saúde, estilos de vida e doenças crônicas. Brasil, grandes regiões e unidades da federação. Rio de Janeiro: IBGE, 2014. Disponível em: http://portalarquivos.saude.gov.br/images/pdf/2014/dezembro/18/PNS-2013.pdf. Acesso em: 10 jul. 2019.

IBGE - Instituto Brasileiro de Geografia e Estatística, 2017. Retrieved from https://cidades.ibge.gov.br/brasil/sp/tupa/panorama. Acesso em: 27 jan. 2019.

KNIGHT, D. A., THOMPSON, D., MATHIE, E., DICKINSON, A. 'Seamless care? Just a list would have helped!' Older people and their carer's experiences of support with medication on discharge home from hospital. Health Expect, Malden, v. 16, n. 3, p. 277-91, set. 2013.

MARCONI, M. A., LAKATOS, E. M. Técnicas de pesquisa. In: MARCONI, M. A.; LAKATOS, E. M. (eds.). Fundamentos de metodologia científica. 7. ed.. São Paulo: Atlas. 2010. p. 157197.

MINAYO, M. C. S. O desafio do conhecimento: pesquisa qualitativa em saúde. 13. ed. São Paulo: Hucitec. 2013.

MOEN, J. et al. "I don't know how many of these [medicines] are necessary": a focus group study among elderly users of multiple medicines. Patient Educ Couns, Oslo, v. 74, n. 2, p. 13541, feb. 2009.

MORAES, G. V. O. Influência do saber biomédico na percepção da relação saúde/doença/ incapacidade em idosos na comunidade. 2012. Dissertação (Mestrado em Ciências da Saúde) Centro de Pesquisas René Rachou, FIOCRUZ, Belo Horizonte, 2012.

MORAES, G. V. O. et al. O. A percepção dos idosos sobre o saber biomédico no cuidado à velhice e às "coisas da idade", Physis - Revista de Saúde Coletiva, Rio de Janeiro, v. 26, n. 1, p. 309-329, 2016.

MUNIZ, E. C. S. et al. Análise do uso de medicamentos por idosos usuários de plano de saúde suplementar. Rev Bras Geriatr Gerontol., Rio de Janeiro, v. 20, n. 3, p. 375-387, 2017.

OLIVEIRA, L. P. B. A, SANTOS, S. M. A. a. Conciliando diversas formas de tratamento à saúde: um estudo com idosos na atenção primária. Texto Contexto Enferm, [S.I.] v. 25, n. 3, e:3670015, 2016a. Disponível em: https://www.redalyc.org/pdf/714/71446759025.pdf. Acesso em: 22 jan. 2019.

OLIVEIRA, L. P. B. A, SANTOS, S. M. A. b. Uma revisão integrativa sobre o uso de medicamentos por idosos na atenção primária à saúde. Rev Esc Enferm USP., São Paulo, v. 50, n. 1, p. 167-179, 2016 b.

PAULA JÚNIOR, J. D. P. et al. Prática de polifarmácia por idosos cadastrados em unidade de 
atenção primária. Investigação, Franca, v. 13, p. 15-18, 2013. Disponível em:

http://publicacoes.unifran.br/index.php/investigacao/article/view/796. Acesso em: 17 jan. 2019.

PEREIRA, K.G. et al. Polifarmácia em idosos: um estudo de base populacional. Rev Bras Epidemiol, São Paulo, v. 20, n. 2, p. 335-344, abr./jun. 2017.

RAMOS, L. R., et al. Polimefarmácia e polimorbidade em idosos no Brasil: um desafio em saúde pública. Rev Saúde Pública, São Paulo, v. 50, p. 1-13, 2016. Disponível em: http://www.scielo.br/pdf/rsp/v50s2/pt_0034-8910-rsp-s2-S01518-87872016050006145.pdf. Acesso em: 12 jan. 2019.

SALES, A. S.; SALES, M. G. S.; CASOTTI, C. A. Perfil farmacoterapêutico e fatores associados à polimedicação entre idosos de Aiquara, Bahia, em 2014. Epidemiol Serv Saude, Brasília, v. 26, n. 1, p. 121-32, 2017. Disponível em: http://www.scielo.br/pdf/ress/v26n1/2237-9622-ress26-01-00121.pdf. Acesso em: 20 jan. 2019.

SANTOS, T. R. A. et al. Consumo de medicamentos por idosos, Goiânia, Brasil. Rev Saúde Pública, São Paulo, v. 47, n. 1, p. 94-103, 2013. Disponível em: http://www.scielo.br/pdf/rsp/v47n1/13.pdf. Acesso em: 17 dez. 2018.

SILVA, C.V. et al. Representações sociais de idosos sobre a polifarmácia. In: CONGRESSO IBERO-AMERICANO EM INVESTIGAÇÃO QUALITATIVA, 7., 2018, Fortaleza. Atas... Aveiro: Ludomedia, 2018. p. 707-716.

SILVA, A. E., MACEDO, C. L. Polifarmácia em idosos. Revista Saúde e Pesquisa, Maringá, v. 6, n. 3, p. 477-486, set./dez. 2013.

SILVA, S. P. C., MENANDRO, M. C. S. As representações sociais da saúde e de seus cuidados para homens e mulheres idosos. Saúde Soc., São Paulo, v. 23, n. 2, p. 626-640, 2014.

SILVA, R., SCHMIDT, O. F., SILVA S. Polifarmácia em geriatria. Rev AMRIGS, Porto Alegre, v. 56, n. 2, p. 164-74, abr./jun. 2012.

SILVA, C. H., SPINILLO, C. G. Dificuldades e estratégias no uso de múltiplos medicamentos por idosos no contexto do design da informação. Estudos em Design Revista, Rio de Janeiro, v. 24, n. 3, p. 130-144, 2016. Disponível em:

https://www.eed.emnuvens.com.br/design/article/view/377/253. Acesso em 11 jan. 2019.

SOUSA, S., et al. Polimedicação em doentes idosos: adesão à terapêutica. Rev Port Clínica Geral, Lisboa, v. 27, p. 176-82, 2011.

WHILE, C. et al. Medication management: the perspectives of people with dementia and family carers. Dementia, Londres, v. 12, n. 6, p. 734-50, nov. 2012.

Recebido em: 01 de março de 2019.

Aceito em: 14 de julho de 2019. 